\title{
Experimental tests of sex determination in Goniozus nephantidis (Hymenoptera: Bethylidae)
}

\author{
JAMES M. COOK \\ Department of Biology, Imperial College at Silwood Park, Ascot SL5 7PY, U.K.
}

\begin{abstract}
Single locus complementary sex determination (CSD) occurs in several species of Hymenoptera. Individuals that carry two different alleles (heterozygotes) are female while those with one and two copies of the same allele (hemizygotes and homozygotes) are haploid and diploid males, respectively. A multilocus model, in which diploids are only male if homozygous at all sex loci, has been proposed for species with regular but not exclusive inbreeding. To date, the only explicit test of the multilocus model is for Nasonia vitripennis. The latter is an inbreeding species in the Infraorder Parasitica and was shown not to conform to the multilocus model. In this study, inbreeding experiments are used to refute single and multilocus sex determination in a second inbreeding hymenopteran, Goniozus nephantidis (Infra-order Aculeata). Single locus CSD has been demonstrated in five other aculeates and G. nephantidis is the first aculeate species shown not to have CSD. The absence of CSD in G. nephantidis is probably a derived condition, favoured by the penalty of increased diploid male production under inbreeding.
\end{abstract}

Keywords: Bethylidae, diploid males, Hymenoptera, inbreeding, parasitoid, sex determination.

\section{Introduction}

Whiting (1943) showed that sex determination in the parasitoid wasp Bracon hebetor (Braconidae) depends on the complementary action of multiple alleles at a single major sex locus. Fertilized eggs are diploid and develop as females if heterozygous at the sex locus, but as males if homozygous at the sex locus. Unfertilized eggs are haploid and always develop as males. Diploid males are effectively sterile as they produce diploid sperm (MacBride, 1946). This system of single locus complementary sex determination (CSD) has also been demonstrated in another parasitoid wasp Diadromus pulchellus (Periquet et al., 1993), as well as the bees Apis mellifera (Mackensen, 1951; Woyke, 1965) and A. cerana (Woyke, 1979; Hoshiba et al., 1981) and the sawfly Athalia rosae (Naito \& Suzuki, 1991). It appears that single locus CSD also operates in the sawfly Neodiprion nigroscutum (Smith \& Wallace, 1971), the fire ant Solenopsis invicta (Hung et al., 1972, 1974; Hung \& Vinson, 1976; Ross \& Fletcher, 1985, 1986) and the stingless bee Melipona quadrifasciata (Camargo, 1979), although data from these species do not strictly exclude a multilocus model (Cook, 1993). Diploid males have been detected in more than 20 other species of Hymenoptera, comprising ants, bees and wasps, but comprehensive tests of single locus CSD have not been carried out in these species (Cook, 1993)

It is clear that single locus CSD does not apply to all Hymenoptera as prolonged inbreeding does not lead to diploid male production in some species of parasitoid wasps in the superfamily Chalcidoidea (e.g. Muscidifurax raptor and M. zaraptor: Legner, 1979; Mellittobia sp. 'c': Schmieder \& Whiting, 1947; Nasonia vitripennis: Skinner \& Werren, 1980). Sib-mating is prevalent in many species of parasitoid wasps, notably in the Chalcidoidea, and several authors (e.g. Whiting, 1945; Crozier, 1971; Bull, 1981) have noted that single locus CSD is unlikely to operate in such species, as it would produce large numbers of sterile diploid males. Crozier (1971) suggested that sex in inbreeding species might still depend on heterozygosity, but at multiple loci. Snell (1935) had earlier hypothesized a two locus model for Bracon and Crozier (1971) extended this to multiple loci, each with two or more alleles. According to Crozier's (1971) model, heterozygosity at one or more of the sex loci leads to female development. Because a diploid must be homozygous at several loci to develop 
as a male, several generations of inbreeding may be required to cause appreciable diploid male production. Crozier $(1971,1977)$ argued that under multilocus CSD diploid males might remain rare in inbreeding family lines if occasional outcrosses restored heterozygosity. Askew (1968) argued that populations of many species of parasitoid wasps (especially Chalcidoidea) consist of semi-isolated family lines that occasionally outcross.

Crozier (1971) proposed multilocus CSD as a general model of sex determination in the Hymenoptera and considered that single locus CSD could be subsumed as a special case. While the single locus model readily yields quantitative predictions of diploid male production under inbreeding, the multilocus model is more difficult to test as an unknown number of loci are involved and it is not clear how many generations of inbreeding are required. Multilocus CSD has yet to be demonstrated but it should be noted that multigeneration inbreeding experiments have been performed in very few species (Cook, 1993).

In this paper I report tests of single and multilocus CSD models in Goniozus nephantidis (Bethylidae), a gregarious ectoparasitoid of a number of microlepidopteran larvae in the Indian subcontinent (Cock \& Perera, 1987; Perera et al., 1988). G. naphantidis lays clutches of 5-20 eggs, with clutch size strongly influenced by host size (Hardy et al., 1992). Broods are highly female-biased (Ramachandra Rao \& Cherian, 1927), probably because of local mate competition (Hamilton, 1967), and typically comprise one male plus several females. Most matings occur within the brood (Hardy et al., 1992), leading to considerable inbreeding. However, approximately one-third of broods contain no males at eclosion (J. M. Cook \& I. C. W. Hardy, unpublished data), suggesting that some outbreeding also occurs. These reproductive patterns suggest $G$. nephantidis as a candidate for Crozier's (1971) multilocus model of sex determination.

Although categorized as a parasitoid on the basis of its lifestyle, $G$. nephantidis is in the superfamily Bethyloidea of the Infra-order Aculeata, whereas most parasitoid wasps are classified in the Infra-order Parasitica. Amongst aculeates, there is strong evidence for CSD in five bee species (Apidae) and diploid males have been reported in 11 other aculeate species, including ants, bees and vespid wasps (Cook, 1993). To date, CSD has not been ruled out for any species of Aculeata.

\section{Experimental design}

Both Whiting's single locus and Crozier's multilocus CSD models predict that diploid male production will increase with inbreeding, as the latter increases homozygosity. However, the involvement of multiple loci in Crozier's model requires more generations of inbreeding to generate detectable diploid male production. Diploid male production may manifest itself as a simple increase in the offspring sex ratio (proportion males) or, if diploid males have low viability as in Bracon (Whiting, 1961), may also involve increased offspring mortality.

One consequence of haplodiploidy is that a virgin female can produce haploid male offspring and subsequently mate with them. In genetic terms, this is equivalent to self-fertilization and results in an isofemale line with no more than two alleles per locus. If strict sibmating is maintained in subsequent generations, heterozygosity is lost at a rapid, known rate. Under single locus CSD the mother $(A i A j)$ mates with a son that carries one of her sex alleles $(A i$ or $A j)$ so 50 per cent of the diploid offspring are male. Diploid male production does not increase in subsequent generations as the isofemale line cannot lose another sex allele or it will become extinct. In contrast, if sex determination depends on more than one locus, diploid male production increases with each generation of inbreeding until all but one sex locus is fixed. At this time, the multilocus system has effectively collapsed to a single locus system with two alleles. The number of generations required for this to happen provides an estimate of the number of sex loci that were initially heterozygous.

While sex ratio and/or mortality changes provide the main test of diploid male production, increases in the number of all-male broods can also indicate diploid males as diploid males are sterile and their mates will produce only unfertilized (haploid male) eggs.

\section{Materials and methods}

\section{Animals}

The flour moth Corcyra cephalonica was used as the host species for $G$. nephantidis. A regular supply of Corcyra cephalonica larvae was maintained as follows. Once a week, approximately 30 adult moths were placed into each of two large glass sweet jars $(12 \mathrm{~cm}$ diameter, $28 \mathrm{~cm}$ deep) containing a $6 \mathrm{~cm}$ deep layer of culture medium. The medium was a $7: 3$ mixture of wheatgerm and maize meal, with $100 \mathrm{ml}$ glycerol per $5 \mathrm{~kg}$ dry medium and a tablespoon of dry yeast. The tops of jars were covered with filter papers and the jars kept in darkness at $30^{\circ} \mathrm{C}$ and 70 per cent r.h. Suitably sized larvae were available for experiments after about 3 weeks at these conditions. 
Mated female wasps were placed individually in glass tubes ( $2.5 \mathrm{~cm}$ diameter, $8 \mathrm{~cm}$ depth), containing a single host caterpillar and plugged with cotton wool. The experiments were carried out in a controlled environment room at $30^{\circ} \mathrm{C}$ and 70 per cent r.h. with a 16 L:8 D photoperiod. The parasitoids originated from cultures at the Coconut Research Institute, Lunuwila, Sri Lanka.

\section{Experiment 1}

An isofemale line was created as follows. A single, unmated female wasp was placed in a glass tube with a host caterpillar and a drop of 50 per cent honey solution. She produced a brood of (haploid) males. When the brood eclosed, the mother was paired with one of her (haploid) sons and placed in a glass tube with a host and a drop of 50 per cent honey solution. This mother/ son mating ensured a maximum of two sex alleles per locus in the resulting brood. The brood from the mother/son cross comprised one male plus eight females. Sib-mating occurred within this brood and, approximately 1 day after the emergence of the last $F_{1}$ female, each $F_{1}$ female was removed to a new glass tube containing a single host larva (weighing 30-40 $\mathrm{mg}$ ) and a drop of 50 per cent honey solution. The broods laid by these mated $F_{1}$ females formed the first experimental generation. When broods reached the pupal stage the mother was removed (this does not affect brood survival; Hardy \& Blackburn, 1991). The numbers of males and females emerging in each brood were recorded.

Two of the eight $F_{1}$ females failed to reproduce. The other six produced one all-female brood, one all-male brood and four broods consisting of one male plus several females. Females from the latter four broods were used to form the next generation. In subsequent generations approximately 20 female wasps were taken from broods containing one male plus several females and used to produce the next generation. The experiment was continued for 22 generations of strict sib-mating.

\section{Experiment 2}

After 22 generations of inbreeding, an experiment was carried out to compare the size, sex ratio and survival of experimentally inbred broods with broods taken from a culture maintained under the same conditions, but with frequent outcrossing. Thirty-five females from mixed sex broods were taken from each stock. Each female was placed in a small petri dish $(6 \mathrm{~cm}$ diameter), containing a single host (weighing $30-40 \mathrm{mg}$ ) and a drop of 50 per cent honey solution. The broods were inspected daily and the numbers of eggs, larvae and pupae recorded to obtain a mortality schedule for each stock. Following eclosion the sex ratio of each brood was recorded.

\section{Analysis}

The data were analysed using generalized linear models in the form of the GLIM statistical package (Numerical Algorithms Group, Oxford). GLIm permits the analysis of proportional data (e.g. sex ratio, mortality) without transformation, by specifying a binomial error distribution. When binomial errors are specified, the model is fitted using the logit (log odds) of the response variable. The significance of explanatory variables is assessed by fitting a full statistical model and then subtracting elements of the model. The change in deviance produced when a variable is removed is a measure of its significance (McCullagh \& Nelder, 1983). When errors are normally distributed (e.g. brood size), analysis of deviance equals analysis of variance and $F$ and $r^{2}$ values can be calculated. When errors are binomially distributed, the change in deviance approximates to a chi-square distribution (McCullagh \& Nelder, 1983). In models assuming binomial error distributions, a heterogeneity factor (h.f.) is calculated. This equals the residual deviance divided by the degrees of freedom when the full model is fitted. If the data are distributed exactly as predicted by the binomial model, h.f. $=1$. Values of h.f. much greater than one indicate overdispersion of the data and invalidate model assumptions. In practice, h.f. values of about three or more can be taken to indicate that the error structure is inappropriate and that the data require transformation (McCullagh \& Nelder, 1983).

Standard sized hosts were used in the experiments because $G$. nephantidis lays more eggs on larger hosts (Hardy et al., 1992). As insufficient standard sized hosts were available in generations 6,12 and 15-18 of experiment 1 , the data from these generations were excluded from brood size and sex ratio analyses. Data from all generations were used to test the hypothesis that the frequency of all-male broods increases with inbreeding. In both experiments, hosts weighing 30-40 mg were used in an attempt to control for host size effects. Residual host weight effects were tested for by including host weight as a covariate in each model.

\section{Results}

\section{Experiment 1}

Figure 1 displays mean brood sex ratios $( \pm 1$ S.E.) in successive generations of inbreeding. A full statistical model (generation + host weight) was fitted first, 


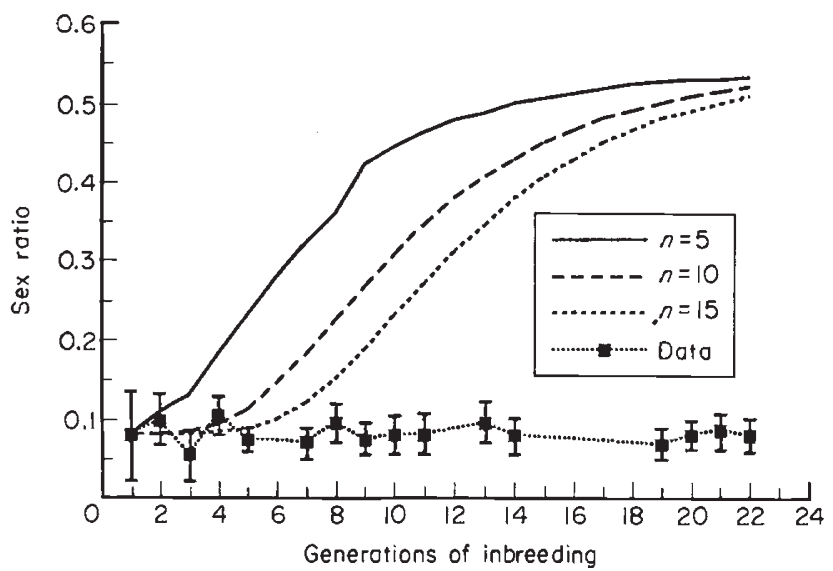

Fig. 1 Mean (eclosion) brood sex ratios (with S.E.) for $G$. nephantidis and predicted values for 5, 10 and 15 locus CSD systems under experimental inbreeding. The method for calculating expected values is given in the Appendix.

assuming binomial error variance. Regressions of sex ratio on host weight (chi-squared $=0.16$, d.f. $=1$, $P>0.1$, h.f. $=0.61$ ) and generations (chi-squared $=0.16$, d.f. $=1, P>0.1$, h.f. $=0.61$ ) were not significant and both variables accounted for less than 0.1 per cent of the deviance.

Mean brood sex ratio was highly female-biased at $0.076 \pm 0.005$ and no broods contained more than two males. The less than binomial variation (h.f. $=0.61$ ) in sex ratio occurs because about 70 per cent of broods have exactly one male and few have two or no males. This pattern is also found in other Goniozus spp. and indicates a considerable degree of precision in the allocation of sex to the offspring in a brood (Green et al., 1982). Selection favours such precise sex allocation because mating normally occurs among siblings in a brood and females developing in broods without males have little chance of insemination (Hartl, 1971).

Host size has a significant effect on brood size $(F=4.42$, d.f. $=1,300,0.01<P<0.05)$ but the slope is only just significantly greater than zero $(t=2.1)$ and the model accounts for only 1.5 per cent of the deviance. The regression of brood size on generations, after controlling for host size, is not significant $(F=0.00$, d.f. $=1,299, P>0.05)$. Only 15 of 398 broods were allmale. Regression of the proportion of all-male broods on generations is not significant (chi-squared $=0.96$, d.f. $=1, P>0.1$, h.f. $=1.85$ ) and explains only 2.65 per cent of the deviance.

In summary, inbreeding for 22 generations produced no significant changes in brood size, brood sex ratio or the frequency of all-male broods.

\section{Experiment 2}

Thirty of thirty-five inbred broods and 31 of 35 partially outbred broods contained both male and female offspring. The mean sex ratio was $0.08 \pm 0.012$ and the heterogeneity factor was 0.57 , indicating less than binomial variance. Brood sex ratio did not vary between stocks (chi-squared $=0.01$, d.f. $=1$ ) and the regression of brood sex ratio on host weight (chisquared $=0.26$, d.f. $=1$ ) was not significant. Mean egg to adult survival was $0.87 \pm 0.013$ and did not vary significantly between inbred and outbred broods (chisquared $=0.00$, d.f. $=1$, h.f. $=1.56$ ) and the regression of survival on host weight was not significant (chisquared $=0.03$, d.f. $=1$ ).

\section{Discussion}

\section{Tests of multilocus CSD}

The data collected provide no evidence of diploid male production and favour rejection of CSD as the mode of sex determination in $G$. nephantidis. However, before accepting this conclusion, two points must be considered. First, complete homozygosity would be required to test a multilocus CSD model that invokes a large but unspecified number of loci. However, selection would be too weak to maintain heterozygosity at many loci, unless they had pleiotropic effects (Crozier, 1971, 1977; Bull, 1981). This is because, for a system with $n$ loci, each with two alleles, the probability of a diploid being homozygous at all loci (and therefore male) is $0.5^{\mathrm{n}}$. For example, if $n=10$, only about 1 in 1000 fertilized eggs is a diploid male. For this reason, I consider that exclusion of CSD systems involving up to 10 loci can be taken as strong refutation of the multilocus model.

In general, the number of loci at which alleles are fixed increases with the number of generations of inbreeding. After $t$ generations, it is possible to say that diploid male production should be detectable in any CSD system involving $n$ or less loci. Calculation of expected levels of diploid male production is relatively complex; the general method is explained in the Appendix. Figure 1 shows the Goniozus sex ratio data alongside expected sex ratio changes (owing to increases in diploid male production) for 5, 10 and 15 locus CSD systems. The conclusion is that the inbreeding procedure would have revealed diploid male production even with 15 heterozygous sex loci, a number far greater than has been suggested. In fact, 10 generations of inbreeding would be more than adequate to test even a 15 locus model. 


\section{Inbred experimental populations}

A second and important problem relates to experiments on insects from laboratory populations. The latter are often highly inbred and may have very limited genetic variability. Population bottlenecks, such as limited initial field collections and population crashes in laboratory cultures, could result in a population with only two sex alleles at a single locus. In fact, Petters \& Mettus (1980) argued that many laboratory populations of $B$. hebetor may be in this state. In the single locus, two allele model (SLTAM) further inbreeding does not increase the proportion of diploid males as this has already reached its upper limit $(50$ per cent of diploids). CSD cannot therefore be detected via sex ratio or mortality changes. This problem has not been previously addressed but may apply to many species in which CSD is likely to be investigated. I show below that it is possible to exclude SLTAM for G. nephantidis, by showing that the highly female-biased brood sex ratio and low brood mortality is inconsistent with the SLTAM requirement that 50 per cent of fertilized eggs are male.

The parasitoids used in my experiments originated from a 10-year-old culture. Although this had been periodically augmented with wild-stock, it is still possible that the experimental population had one sex locus with two alleles. If $a$ is the proportion of unfertilized eggs, then, the primary sex ratio $\left(r_{1}\right)$ is

$r_{1}=a+(1-a) / 2$.

$r_{1}$ must lie between 0.5 (if all eggs are fertilized and 50 per cent of fertilized eggs are male) and 1 (all eggs unfertilized - haploid males only). Thus $r_{1}$ cannot be female-biased. The eclosion sex ratio $\left(r_{2}\right)$ depends also on the viability of sex types. As diploid males may have reduced viability (Whiting, 1961; Petters \& Mettus, 1980 ), let their viability be $v$ relative to normal males and females. Then $r_{2}$ is calculated as follows:

$r_{2}=\frac{a+v(1-a) / 2}{1-(1-v)(1-a) / 2}$.

While $r_{1}$ cannot be female-biased, differential mortality of diploid males can lead to female bias in $r_{2}$. In fact any value of $r_{2}$ can be consistent with the single locus, two allele model (SLTAM) but female bias is only possible if there is differential mortality of diploid males. Consequently, sLTAM can be excluded if brood survival is too high to explain a female bias via differential mortality of diploid males (Fig. 2).

SLTAM is tested by comparing observed brood survival with the value predicted assuming that all eggs are fertilized $(a=0)$. This is an unlikely scenario but consti-

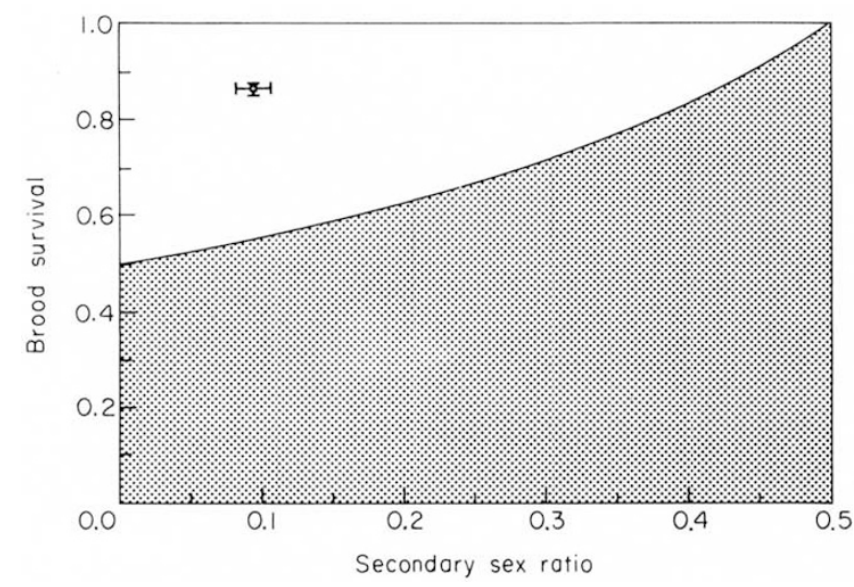

Fig. 2 The use of brood survival and secondary sex ratio data to test SlTAM. For a given secondary sex ratio, any survival value falling in the shaded area is consistent with SLTAM. Points falling outside the shaded area are inconsistent with SLTAM because there is insufficient mortality to generate the degree of female bias observed. The mean brood survival and brood sex ratio values ( \pm 1 S.E.) for $G$. nephantidis (from experiment 2) are clearly inconsistent with SLTAM.

tutes the minimum mortality required to cause female bias because it gives the greatest proportion of females at oviposition. The predicted value is obtained by estimating $v$ from the observed $r_{2}$ value and then using $v$ to calculate expected mortality. Substitution of $a=0$ simplifies eqn 2 :

$r_{2}=v /(1+v)$.

Because $r_{2}$ was measured as 0.08 in experiment 2 , $v=0.087$ from eqn 3 .

The denominator of eqn 2 represents the total number of individuals of all sex types reaching maturity, i.e. the brood survival. Because $a=0$, the expression for brood survival $(b s)$ simplifies to:

$b s=(1+v) / 2$.

The value for $v(0.087)$ is now substituted into eqn 4 to yield the maximum brood survival consistent with SLTAM: $b s=1.087 / 2=0.544$. As mean brood survival in experiment 2 was 0.871 (S.E. $=0.013$ ), SLTAM can be rejected at the 1 per cent level for $G$. nephantidis (lower limit of $t$-distribution $=0.84$, see Fig. 2).

The mortality/sex ratio test described can only be applied to species with female-biased sex ratios and measurable mortality. It is also limited in being a oneway test - it cannot establish the presence of diploid males. Nevertheless, it offers a rapid, quantitative test which may be applied easily to many parasitoid species without recourse to cytological studies. The test also draws attention to the need to refute SLTAM to avoid false negative tests of CSD when using laboratory 
populations. In species where this test is either not possible or not conclusive, cytological investigations are recommended.

\section{Evolutionary interpretations}

The occurrence of CSD in both suborders (Symphyta and Apocrita) of the Hymenoptera and in both Infra-orders (Aculeata and Parasitica) of the Apocrita suggests that CSD is ancestral in the Hymenoptera, as proposed by Schmieder \& Whiting (1947) and Crozier (1977). Theoretical consideration of evolutionary transitions between single locus CSD and other sex determination systems further support this view (Bull, 1981).

None of the species so far shown to have CSD experiences regular inbreeding, which increases the production of sterile, diploid males. The life history of $G$. nephantidis suggested it as an appropriate species to test Crozier's (1971) multilocus CSD model for inbreeding species. However, multilocus CSD does not occur in G. nephantidis. Multilocus CSD has also been ruled out for the inbreeding chalcidoid wasp Nasonia vitripennis (Skinner \& Werren, 1980) and is probably not applicable to the (largely inbreeding) superfamily Chalcidoidea.

As single locus CSD is probably ancestral in the Hymenoptera, different sex-determining mechanisms may have evolved in various inbreeding lineages. For example, while G. nephantidis (Aculeata: Bethyloidea) and $N$. vitripennis (Parasitica: Chalcidoidea) share features such as the parasitoid lifestyle and femalebiased sex ratios, each is more closely related to (different) species with single locus CSD than they are to each other. This theme may be further embodied in different super-families of the Infra-order Parasitica, which is polyphyletic (Königsmann, 1978; Hennig, 1981) and includes several lineages that have independently evolved inbreeding systems.

Sex determination in species of Hymenoptera without CSD is not understood. Two possible mechanisms are environmental sex determination via fertilization and sex determination via the ratio of nuclear to cytoplasmic products (Crozier, 1971). The fertilization model has been excluded for $N$. vitripennis (Friedler \& Ray, 1951) but this does not preclude its operation in G. nephantidis or other inbreeding Hymenoptera. The second model could operate in a similar way to Drosophila sex determination: a maternal (da-like) product in the egg could promote expression of a male developmental pathway while nuclear (sis-like) loci act to inhibit the maternal product. The haploid nuclear product is insufficient to stop activation of the male pathway but the diploid does cause the switch to female development.
To date, sex determination experiments have been largely confined to a few taxonomic groups within the Hymenoptera. Only two members of the suborder Symphyta have been studied (Smith \& Wallace 1971; Naito \& Suzuki, 1991). Both of these fall in the superfamily Tenthredinoidea. There have been no studies of sex determination in the other five symphytan superfamilies. In the Infra-order Parasitica of the suborder Apocrita, studies have been largely restricted to species of only two (Ichneumonoidea and Chalcidoidea) of the nine super-families. In the Infra-order Aculeata, several bees (Apidae) have been studied (Mackensen, 1951; Garofalo, 1973; Camargo, 1979; Woyke, 1979; Hoshiba et al., 1981), but G. nephantidis is the only non-Apid aculeate to have received attention and no members of the super-families Vespoidea or Sphecoidea have been studied. Further tests of CSD models will be most interesting if they attempt to redress this taxonomic imbalance.

\section{Acknowledgements}

I am grateful to Charles Godfray, Sally Power, Hefin Jones, Ross Crozier and Susan Lawler for comments on various versions of this manuscript. The research was conducted at Imperial College, Silwood Park while I was in receipt of a SERC Research Studentship.

\section{References}

ASKEW, R. R. 1968. Considerations on speciation in the Chalcidoidea (Hymenoptera). Evolution, 22, 642-645.

BULL, J. J. 1981. Coevolution of haplodiploidy and sex determination in the Hymenoptera. Evolution, 35, 568-580.

CAmargo, C. A. 1979. Sex determination in bees. XI. Production of diploid males and sex determination in Melipona quadrifasciata (Hymenoptera, Apidae). J. Apic, Res., 18, 77-84.

COCK, M. J. W. AND PERERA, P. A. C. R. 1987. Biological control of Opisina arenosella (Lepidoptera, Oecophoridae). Biocontrol News Informat., 8, 283-310.

CoOK, J. M. 1993. Sex Determination in the Hymenoptera: A review of models and evidence. Heredity, in press.

CROZIER, R. H. 1971. Heterozygosity and sex determination in haplodiploidy. Am. Nat., 105, 399-412.

CROZIER, R. H. 1977. Evolutionary genetics of the Hymenoptera. Ann. Rev. Entomol., 22, 263-288.

FRIEDLER, C. AND RAY, O. T. 1951. Androgenesis in the wasp Mormoniella. Anat. Rec., 111, 475.

GAROFALO, C. A. 1973. Occurrence of diploid drones in a Neotropical bumblebee. Experientia, 29, 726-727.

GREEN, R. F., GORDH, G. AND HAWKINS, B. A. 1982. Precise sex ratios in highly inbred parasitic wasps. Am. Nat., 120, 653-665.

HAMILTON, w. D. 1967. Extraordinary sex ratios. Science, 156, 477-488. 
HARDY, I. C. W. AND BLACKBURN, T. M. 1991. Brood guarding in a bethylid wasp. Ecol. Entomol., 16, 55-62.

HARDY, I. C. W., GRIFFITHS, N. T. AND GODFRAY, H. C. J. 1992. Clutch size in a parasitoid wasp: a manipulation experiment. $J$. Anim. Ecol., 61, 121-129.

HARTL, D. L. 1971. Some aspects of natural selection in arrhenotokous populations. Am. Zool., 11, 309-325.

HENNIG, w. 1981. Insect Phylogeny. John Wiley and Sons, New York.

Hoshiba, H., OKADA, I. AND KUSANAGI, A. 1981. The diploid drone of Apis cerana japonica and its chromosomes. $J$. Apic. Res., 20, 143-147.

HUNG, A. C. F., IMAl, H. T. AND KUBOTA, M. 1972. The chromosomes of nine ant species from Taiwan, Republic of China. Ann. Entomol. Soc. Am., 65, 1023-1025.

HUNG, A. C. F. AND VINSON, S. B. 1976. Biochemical evidence for queen monogamy and sterile male diploidy in the fire ant, Solenopsis invicta. Isozyme Bull., 9, 42.

HUNG, A. C. F., Vinson, S. B. AND SUMMERLin, J. W. 1974. Male sterility in the red imported fire ant, Solenopsis invicta. Ann. Entomol. Soc. Am., 67, 909-912.

KÖNIGSMANN, E. 1987. Das phylogenetische system der Hymenoptera. Teil 3: 'Terebrantes' (Unterordnung Apocrita). Dt. Ent. Z. (n.F.)., 25, 1-55.

LEGNER, E. F. 1979. Prolonged culture and inbreeding effects on reproductive rates of two pteromalid parasites of muscoid flies. Ann. Entomol. Soc. Am., 72, 114-118.

MACBRIDE, D. H. 1946. Failure of sperm of Habrobracon diploid males to penetrate the eggs. Genetics, 31, 224.

MACKENSEN, o. 1951. Viability and sex determination in the honey bee (Apis mellifera). Genetics, 36, 500-509.

MCCUllaGH, P. AND NELDER, J. A. 1983. Generalised Linear Models. Chapman and Hall, London.

NAITO, T. AND SUZUKI, H. 1991. Sex determination in the sawfly, Athalia rosae ruficornis (Hymenoptera): Occurrence of triploid males. J. Hered., 82, 101-104.

PERERA, P. A. C. R., GOdFraY, H. C. J. AND HASSELl, M. P. 1988. Population dynamics of the coconut caterpillar, Opisina arenosella Walker (Lepidoptera: Xylorictidae), in Sri Lanka. Bull. Entomol. Res., 78, 479-492.

PERIQUET, G., HEDDERWICK, M. P., EL AGOZE, M. AND POIRIE, M. 1993. Sex determination in the Hymenoptera Diadromus pulchellus (Ichneumonidae): validation of the one-locus multi-allele model. Heredity, 70, 420-427.

PETTERS, R. M. AND METTUS, R. V. 1980. Decreased diploid viability in the parasitoid wasp, Bracon hebetor. J. Hered., 71, 353-356.

RAMACHANDRA RAO, Y. AND CHERIAN, M. C. 1927. Notes on the life history and habits of Perisieriola spp. the Bethylid parasite of Nephantis serinopa. Yearbook Dept. Agric. Madras, 11, 11-22.

ROSS, K. G. AND FLETCHER, D. J. C. 1985. Genetic origin of male diploidy in the fire ant, Solenopsis invicta, and its evolutionary significance. Evolution, 39, 888-903.

RoSS, K. G. AND FLETCHER, D. J. C. 1986. Diploid male production - a significant colony mortality factor in the fire ant, Solenopsis invicta (Hymenoptera: Formicidae). Behav. Ecol. Sociobiol., 19, 283-291.

SCHMIEDER, R. G. AND WHITING, P. W. 1947. Reproductive economy in the chalcidoid wasp Melittobia. Genetics, 32, 29-37.
SKINNER, S. W. AND WERREN, J. H. 1980. The genetics of sex determination in Nasonia vitripennis. Genetics, 94, s98.

SMITH, S. G. AND WALLACE, D. R. 1971. Allelic sex determination in a lower hymenopteran, Neodiprion nigroscutum Midd. Can. J. Genet. Cytol., 13,617-621.

SNELL, G. D. 1935. The determination of sex in Habrobracon. Proc. Natl. Acad. Sci. U.S.A., 21, 446-453.

Whiting, A. R. 1961. Genetics of Habrobracon. Adv. Genet., 10, 333-406.

WHITING, P. W. 1943. Multiple alleles in complementary sex determination of Habrobracon. Genetics, 28, 365-382.

Whiting, P. w. 1945 . The evolution of male haploidy. Q. Rev. Biol., 20, 231-260.

WOYKE, J. 1965. Genetic proof of the origin of diploid drones from fertilized eggs of the honeybee. J. Apic. Res., 4, 7-11. woyke, J. 1979. Sex determination in Apis cerana indica. J. Apic. Res., 18, 122-127.

\section{Appendix}

A four step process is used to calculate the expected effect of inbreeding on the sex ratio for a species with multilocus CSD, involving $n$ independent loci. First, the loss of heterozygosity at individual loci is modelled. Second, combinations of $n$ independent loci are considered. Next, the sex determination loads (proportion of diploids that are male) produced by these combinations are calculated. Finally, the proportion of fertilized and unfertilized eggs are added to the equation to yield sex ratio predictions.

\section{Loss of heterozygosity at one locus}

In an inbreeding line resulting from a mother/son mating, any given locus can have only one or two different alleles (barring new mutations). In such a line, only the dimorphic loci are effective in sex determination. Following the mother/son cross, all matings are between siblings. Two types of mating can occur at dimorphic loci: type $\mathbf{X}$ matings occur when the female is heterozygous $\left(A_{i} A_{k} \times A_{i}\right)$ whereas type $\mathbf{Y}$ matings occur when the female is homozygous and the male carries a different allele $\left(A_{i} A_{i} \times A_{k}\right)$. If the locus is monomorphic, all matings are type $\mathbf{Z}\left(A_{i} A_{i} \times A_{i}\right)$.

The loss of heterozygosity at a dimorphic locus is modelled using the probabilities of transitions between different mating types $(\mathbf{X}, \mathbf{Y}$ or $\mathbf{Z})$ in generations $t$ and $t+1$. The system can then be described by three recurrence equations (one for each mating type), which can be iterated to track the loss of heterozygosity with time:

$$
\begin{aligned}
& \mathbf{X}_{\mathrm{t}+1}=0.5 \mathbf{X}_{\mathrm{t}}+\mathbf{Y}_{\mathrm{t}} \\
& \mathbf{Y}_{\mathrm{t}+1}=0.25 \mathbf{X}_{\mathrm{t}} \\
& \mathbf{Z}_{\mathrm{t}+1}=0.25 \mathbf{X}_{\mathrm{t}}+\mathbf{Z}_{\mathrm{t}} .
\end{aligned}
$$




\section{Several independent loci}

The above equations give the probability of any initially dimorphic locus being in state $\mathbf{X}, \mathbf{Y}$ or $\mathbf{Z}$ after $t$ generations. As Crozier's model specified independent loci, the fixation probability of each sex determination locus does not depend on any other sex locus. Using the multinomial probability theory, it is possible to calculate the probabilities of all combinations of $\mathbf{X}, \mathbf{Y}$ and $\mathbf{Z}$ for $n$ independent loci. After $t$ generations, the probability that $x$ loci are in state $\mathbf{X}, y$ in state $\mathbf{Y}$ and $z$ in state $\mathbf{Z}$ is:

$P_{\mathrm{XYZ}}=\frac{n !}{x ! y ! z !} P_{\mathrm{X}}^{\mathrm{x}} P_{\mathrm{Y}}^{\mathrm{y}} P_{\mathrm{Z}}^{\mathrm{z}}$.

\section{Sex determination loads}

For any number of loci $n$, after $t$ generations, many combinations of locus states are possible. Each combination leads to a predicted sex determination load, which may or may not be unique to that particular combination. The expected sex determination load for an $n$ locus system, after $t$ generations is therefore calculated as follows. First, the sex determination loads are calculated for each possible combination of locus states. These are then weighted by their probability of occurrence and summed.

If any of the loci are in state $\mathbf{Y}$, all diploid offspring are heterozygous and therefore no diploid males occur. If no loci are in state $\mathbf{Y}$ but a fraction $(\mathrm{x})$ of the loci are in state $\mathbf{X},(1 / 2)^{\mathrm{x}}$ of diploid offspring are male. No other combinations produce diploid males. After summing the weighted sex determination loads, the figure must be divided by a correction factor, which excludes the combinations of locus states that imply matings between diploid and haploid males. These instances are possible because of the matrix structure but obviously do not occur experimentally. The correction factor includes all combinations of loci consisting only of state $\mathbf{Y}$ and/or state $\mathbf{Z}$.

To calculate the sex determination loads in Fig. 1, it was assumed that one-half of the dimorphic loci were initially in state $\mathbf{X}$ and one-half in state $\mathbf{Y}$. Assumptions about initial locus states only alter predictions significantly in the first two or three generations. Subsequently, their influence on predictions is insignificant in comparison to the number of loci involved.

\section{Fertilization rate}

The primary sex ratio depends on both the sex determination load (fertilized eggs may be male or female) and the fertilization rate (unfertilized eggs are always male). If the sex determination load is $b$ and a proportion $(a)$ of eggs are unfertilized, the primary sex ratio $\left(r_{1}\right)$ is: $r_{1}=a+b(1-a)$.

In outbred broods (prior to experimental inbreeding) there are few if any diploid males and the sex ratio provides an estimate of the fertilization rate. In the case of Goniozus nephantidis, where heterozygosity does not affect sex determination, the estimate may be made from inbred or outbred broods. Sex ratio predictions in Fig. 1 were made under the assumption that 0.08 of eggs remain unfertilized, as suggested by the data from experiment 2 . 